OPEN ACCESS

Edited by:

Carmen Sandi,

Ecole Polytechnique Federale De

Lausanne, Switzerland

Reviewed by:

Gernot Riedel,

University of Aberdeen, UK

Carlos Eduardo Neves Girardi, Universidade Federal de Sao Paulo,

Brazil

*Correspondence:

Shigenobu Toda,

Department of Psychiatry and Neurobiology, Kanazawa University

School of Medicine, 13-1 Takara-machi, Kanazawa 920-8641,

Japan

todas@med.kanazawa-u.ac.jp

Received: 21 December 2014 Accepted: 26 April 2015 Published: 13 May 2015

Citation:

Iguchi Y, Kosugi S, Lin Z, Nishikawa H, Minabe $Y$ and Toda S (2015)

Pre-stress performance in an

instrumental training predicts post-stress behavioral alterations in chronically stressed rats.

Front. Behav. Neurosci. 9:119.

doi: 10.3389/fnbeh.2015.00119

\section{Pre-stress performance in an instrumental training predicts post-stress behavioral alterations in chronically stressed rats}

\author{
Yoshio Iguchi ${ }^{1}$, Sakurako Kosugi ${ }^{1}$, Ziqiao Lin ${ }^{1}$, Hiromi Nishikawa ${ }^{1}$, Yoshio Minabe ${ }^{1,2}$ \\ and Shigenobu Toda ${ }^{1,2 *}$ \\ ${ }^{1}$ Department of Psychiatry and Neurobiology, Kanazawa University School of Medicine, Kanazawa, Japan, ${ }^{2}$ Research \\ Center for Child Mental Development, Kanazawa University, Kanazawa, Japan
}

Stress is a major factor in the development of major depressive disorder (MDD), but few studies have assessed individual risk based on pre-stress behavioral and cognitive traits. To address this issue, we employed appetitive instrumental lever pressing with a progressive ratio $(\mathrm{PR})$ schedule to assess these traits in experimentally naïve Sprague-Dawley rats. Based on four distinct traits that were identified by hierarchical cluster analysis, the animals were classified into the corresponding four subgroups (Low Motivation, Quick Learner, Slow Learner, and Hypermotivation), and exposed to chronic unpredictable stress (CUS) before monitoring their post-stress responses for 4 weeks. The four subgroups represented the following distinct behavioral phenotypes after CUS: the Low Motivation subgroup demonstrated weight loss and a late-developing paradoxical enhancement in PR performance that may be related to inappropriate decision-making in human MDD. The Quick Learner subgroup exhibited a transient loss of motivation and the habituation of serum corticosterone (CORT) response to repeated stress. The Slow Learner subgroup displayed resistance to demotivation and a suppressed CORT response to acute stress. Finally, the Hypermotivation subgroup exhibited resistance to weight loss, habituated CORT response to an acute stress, and a long-lasting amotivation. Overall, we identified causal relationships between pre-stress traits in the performance of the instrumental training and post-stress phenotypes in each subgroup. In addition, many of the CUS-induced phenotypes in rats corresponded to or had putative relationships with representative symptoms in human MDD. We concluded that the consequences of stress may be predictable before stress exposure by determining the pre-stress behavioral or cognitive traits of each individual in rats.

\footnotetext{
Keywords: appetitive instrumental learning, chronic unpredictable stress, individual difference, major depressive
} disorder, pre-stress traits, post-stress phenotypes

\section{Introduction}

Stress may lead to various aversive outcomes, and major depressive disorder (MDD) is one of the most damaging consequences (Collins et al., 2011). The increasing number of MDD patients is imposing a growing worldwide social and economical burden (World Health Organization, 2014), 
and some of these patients are resistant to first-line medications (Rush et al., 2006). Numerous preclinical studies have attempted to identify the molecular and cellular mechanisms that underlie MDD to develop effective therapies (McEwen, 2005; Russo and Nestler, 2013).

However, MDD prevention, diagnosis, or medication of MDD cannot be formulated or standardized for each case due to various individual differences. For example, the responses to stress vary substantially among individuals, including animals and humans. Some may exhibit resilience, whereas others may exhibit MDDlike vulnerability after the same stress exposure (Krishnan et al., 2007). In addition, the effectiveness of antidepressants in treating MDD varies among individuals (Trivedi et al., 2006), while the predominant clinical manifestations, severity, or courses differ markedly among individuals (Rush, 2007), which has prompted physicians to subtype this disorder (Harald and Gordon, 2012). It would be highly beneficial if we could predict the consequence for each individual before exposure to a stress that could trigger MDD. This identification could be useful given the individual differences in causality between individual pre-onset traits and post-onset phenotypes. This causality has been investigated vigorously in human longitudinal studies (Fuhr et al., 2014). The corresponding approach has also been applied to preclinical studies using animal models of other mental disorders, such as addiction (Belin et al., 2011; Saunders and Robinson, 2011; Broos et al., 2012), but very few animal studies in terms of MDD (Stedenfeld et al., 2011; Duclot and Kabbaj, 2013; Rygula et al., 2013).

To investigate the possible relationships between pre-stress traits and both early and late-emerging post-stress MDD-related behaviors in rats, we first measured the motivation and bias of action selection in cost- or effort-based decision-making using instrumental training with a progressive ratio (PR) schedule (Kurniawan et al., 2011; Der-Avakian and Markou, 2012) to establish pre-stress phenotypic subgroups. We then re-tested these subgroups for 4 weeks after chronic unpredictable stress (CUS) exposure to identify the causal relationship between prestress traits and post-CUS phenotypes.

\section{Materials and Methods}

\section{Animals}

All animal procedures were in accordance with Guidelines for Proper Conduct of Animal Experiments (Science Council of Japan, June 2006) and approved by Kanazawa University IACUC. Male Sprague-Dawley rats (Japan SLC, Hamamatsu, Japan) initially weighing $275-300 \mathrm{~g}$ were housed 2 or 3/Plexiglas cage $(38 \times 33.5 \times 17 \mathrm{~cm})$ in a climate-controlled vivarium with a $12 / 12$ $\mathrm{h}$ light-dark cycle (lights on at $08: 45 \mathrm{~h}$ ). Instrumental learning trainings (fixed- and progressive-ratio training, set-shifting, and response reversal learning) were conducted during the light phase.

Abbreviations: ADHD, attention-deficit/hyperactive disorder; ANOVA, analyses of variance; CORT, serum corticosterone; CUS, chronic unpredictable stress; FR, fixed ratio; HPA, hypothalamic-pituitary-adrenal; ITI, inter-trial interval condition; MDD, major depressive disorder; NE, novel environment; PR, progressive ratio.

\section{Apparatus}

Instrumental learning was performed in operant chambers equipped with two retractable levers $(30 \times 24 \times 25 \mathrm{~cm}$; Med associates, St. Albans, VT, USA) in a soundproof room. Stimulus light $(100 \mathrm{~mA}, 28 \mathrm{~V})$ for visual cue discrimination learning was mounted above each lever. Chambers were also equipped with a miniature solenoid-activated valve to deliver $50 \mu \mathrm{l}$ of $0.2 \%(\mathrm{w} / \mathrm{v})$ sodium saccharin dihydrate (Wako Pure Chemical Industries, Osaka, Japan) into a recessed magazine between the two levers, except for set-shifting and response reversal learning in which the magazine was relocated to the wall opposite the levers. One $100 \mathrm{~mA}, 28 \mathrm{~V}$ houselight on the top center of the wall opposite the levers provided ambient illumination.

Cocaine- and novelty-induced behaviors were monitored using three photoelectric actimeters (Panlab, Barcelona, Spain), each comprising a novel environment (NE) of transparent acrylic plastic $(45 \times 45 \times 30 \mathrm{~cm})$ under faint illumination (approximately $0.5 \mathrm{~lx}$ ). The floor was covered with wood chips. Two $16 \times 16-$ infrared beam arrays located diagonally at the NE edge were used to monitor locomotor (horizontal) activity.

\section{Timelines of All Experiments}

In Experiment 1, following 4-day initial training, rats were subjected to the trainings of fixed- (FR; 4 days) and progressiveratio (PR; 7 days) instrumental learning. Then, performance in set-shifting and response reversal learning was examined. 6-9 days later, cocaine-induced behaviors were assessed in a NE. In Experiment 2, animals were exposed to chronic unpredictable stress (CUS) or daily handling as control treatment for 28 days following FR and PR instrumental training. Then, animals were maintained for 28 days (post-CUS period) with weekly behavioral assays. A chronological schema of the entire experimental procedure of Experiment 1 and 2 are depicted in Figures 1A, 3A, respectively.

\section{Time-Constraint Progressive Ratio Instrumental Training}

Experimentally naïve rat groups (Experiment 1: $n=79$; Experiment 2: $n=84$ ) handled daily for 5 days before training were presented with bottles of saccharin solution in their home cages for $24 \mathrm{~h}$ before training to ameliorate neophobia to an unfamiliar reward. Response to one lever was rewarded (active lever), whereas that to the other lever was not (inactive lever). Active and inactive lever positions were counterbalanced across animals. First, rats received a 30-min training session over 4 successive days for apparatus habituation, magazine training, manual shaping, and continuous reinforcement training with the active lever alone following 23.5-h water deprivation. After daily session completion, animals were allowed to drink water for $30 \mathrm{~min}$ in their home cages. Then, the water-restriction schedule was lifted (i.e., ad libitum access to water and food). Animals were then subjected to 4 training sessions (once/day) with both levers and changing lever press:reward ratios of 1:1 (FR-1, session 1), 2:1 (FR-2, session 2), and 5:1 (FR-5, sessions 3 and 4). Each session was terminated after 100 rewards or $30 \mathrm{~min}$. Daily training sessions with a PR schedule for 7 (Experiment 1) or 3 days (Experiment 2) followed after them. The PR schedule was 
A
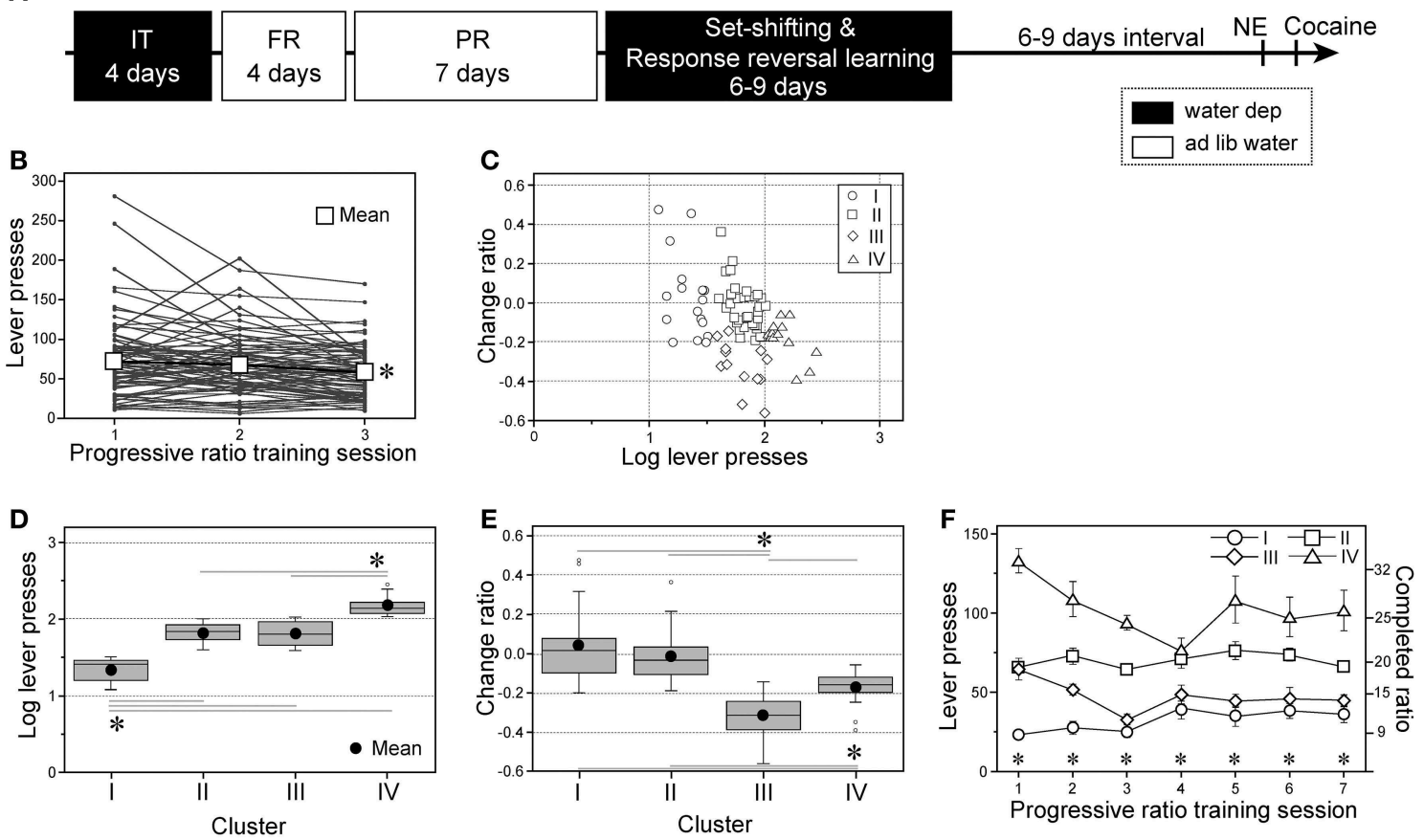

FIGURE 1 | Individual variability in progressive ratio instrumental performance of subgroups. (A) A chronological schema of the entire experimental procedure of Experiment 1. IT, initial instrumental training; FR and $\mathrm{PR}$, fixed- and progressive-ratio instrumental trainings, respectively; NE and cocaine, novel environment- and cocaine-induced behaviors testing, respectively. Black and white boxes = the days with or without water deprivation, respectively. (B) Individual performances with progressive ratio $(\mathrm{PR})$ instrumental training for the first three training sessions. The data of the third session was compared with those of the first and second sessions [session: $\left.F_{(2,156)}=10.01, p<0.0001\right]$ (C) Each rat's performance plotted on a two-factorial surface. $x$-axis: number of lever presses in the first PR session normalized by a common logarithm (log) transformation; $y$-axis: change ratios of lever presses from first $(a)$ to third $(b)$ sessions, $(b-a) /(b+$ a). (D,E) Box plots of the log-transformed lever press numbers during the first PR session; Cluster I, $n=17$; II, $n=36$; III, $n=13$; IV, $n=13$ [D, subgroup: $\left.F_{(3,75)}=106.42, p<0.0001\right]$ and the change ratio $[\mathbf{E}$, subgroup: $\left.F_{(3,75)}=20.58, p<0.0001\right]$. (F) Active lever presses during seven PR sessions by four subgroups. Data are shown as subgroup mean $\pm \mathrm{SEM}$; Cluster I, $n=13$; II, $n=32$; III, $n=12 ;$ IV, $n=10$ [subgroup $\times$ session interaction: $\left.F_{(18,376)}=3.52, p<0.0001\right]$. ${ }^{*} p<0.05$, between sessions (B), between relevant subgroups $(\mathbf{D}, \mathbf{E})$, or significant simple-main effects of subgroup on each session (F). based on the following exponential progression: $1,2,4,6,9,12$, $15,20,25,32,40, \ldots$, derived from the formula $\left[\left(5 \times \mathrm{e}^{0.2 n}\right)-5\right]$ rounded to the nearest integer, where $n$ is the trial number within a session (Richardson and Roberts, 1996). At the start of each daily session, active and inactive levers were inserted into the chamber after a 300-s waiting time, followed by a 35-min long session. Motivation was evaluated as the number of lever presses in each session (and the corresponding highest completed ratio) (Barr and Phillips, 1998).

\section{Set-shifting and Response Reversal Learning}

In Experiment 1, a 23.5-h/day water deprivation schedule was resumed after a 7-day PR training completion, and performance in set-shifting and response reversal learning was examined as described (Floresco et al., 2008; Haluk and Floresco, 2009) with minor modifications. On the day following the last PR session, the magazine was moved to the wall opposite the levers to reform the positional relationship. Animals were retrained on an FR1 schedule to a criterion of 90 reinforcements in $30 \mathrm{~min}$. After familiarization with insertion and retraction of levers, animals were trained to press them within 10-s insertion. A session comprised 90 trials at 20 -s intervals. A trial began with houselight illumination and insertion of either lever into the chamber. Failure of the rat to press the lever within $10 \mathrm{~s}$ resulted in lever retraction and chamber darkening; the trial was scored as an omission. Responses by the rat within $10 \mathrm{~s}$ resulted in lever retraction, an immediate reward, and houselight illumination for another $4 \mathrm{~s}$. Right and left levers were presented once in every pair of trials in a random order. Each animal had to achieve a criterion of 5 or fewer omissions over 90 trials to proceed to set-shifting the next day. In set-shifting, animals were first trained to press a lever with overhead cue light illumination (visual cue discrimination), then to press one particular lever (left or right) consistently while ignoring the visual cue (response discrimination). Retraction of both levers and chamber darkening occurred before each visual cue discrimination training session [=inter-trial interval condition (ITI)]. Every $20 \mathrm{~s}$, a trial began with illumination of the stimulus cue light; $3 \mathrm{~s}$ later, the houselight was turned on, and both levers were inserted into the chamber. Responses to the correct lever (beneath the cue light) resulted in retraction of both levers, a reward, and houselight illumination for another $4 \mathrm{~s}$, after which the chamber returned to ITI. Incorrect responses resulted 
in retraction of both levers and the houselight being turned off immediately without reinforcement. Failure to press either lever within $10 \mathrm{~s}$ was recorded as an omission; the chamber returned to ITI. For each animal, trials continued until a minimum of 30 trials and a criterion performance of 8 consecutive correct choices were achieved. On the following day, response discrimination training commenced. The ITI and criterion of success were identical to those in the visual cue discrimination task, within a maximum of 112 trials.

On the following day of set-shifting, reversal learning commenced. Animals were trained to press the lever resulting in a reward during the preceding set-shifting training (initial response discrimination); then, the position of the correct lever was reversed on the next day. For reversal learning, the ITI condition, houselight illumination, and success/failure criteria were the same as those for set-shifting, except that the cue light was not presented.

Errors made during set-shifting were categorized into subtypes, perseverative, regressive, and never-reinforced, described previously (Floresco et al., 2008; Haluk and Floresco, 2009). Perseverative errors were scored when a rat responded to the lever with a cue light in trials requiring response to the opposite lever. Eight of every 16 consecutive trials required responses in this manner, and these trials were separated into consecutive blocks of 8 trials each. Perseverative errors were scored when a rat pressed the incorrect lever in 6 or more of 8 trials/block. Once a rat made 5 or fewer perseverative errors in a block for the first time, all subsequent errors of this type were no longer counted as perseverative, but instead scored as regressive. Never-reinforced errors were scored when a rat pressed the incorrect lever in trials with cue light illumination above the correct (rewarded) lever. Regressive and never-reinforced errors were used as indices of maintaining and acquiring a new strategy, respectively, and perseverative errors as an index of disengagement from a previously relevant learning rule.

\section{Cocaine-induced Behaviors}

In Experiment 1, the water-restriction schedule was again lifted on set-shifting and response reversal learning completion; 6-9 days later, animals were placed into a NE for 1-h acclimation. On the next day, animals were placed into the NE for $30 \mathrm{~min}$ to monitor spontaneous activity, then injected intraperitoneally with $15 \mathrm{mg} / \mathrm{kg}$ cocaine hydrochloride (Takeda Pharmaceutical Co., Osaka, Japan), and placed back into the NE for $120 \mathrm{~min}$ to assess cocaine-induced behaviors.

\section{CUS Exposure}

In Experiment 2, naive rats were assigned to either the handled (control) or CUS-exposed group after completion of 3 daily PR sessions. The CUS group was housed singly with ad libitum access to food and water and subjected to CUS as described previously (Dias-Ferreira et al., 2009) with some modifications. During the 4 -week period, rats were exposed to 8 cycles of 3 kinds of stressor, restraint, forced swimming, or social defeat (representing psychological, physical, and social stressors, respectively), with 4 stress-free days. One of the three stressors was imposed every day in an unpredictable order at various times during the light phase. Intensities of these stressors were escalated on a weekly basis to prevent habituation. In restraint stress sessions, each animal was immobilized inside a size-fitted, transparent polyethylene terephthalate tube for $30 \mathrm{~min}$ during the first week, 45 min during the second week with simultaneous illumination from a $200-\mathrm{W}$ bulb plus 5 -min rotary shaking, and 60 min during third and fourth weeks with illumination plus 20min shaking. In forced swimming, each animal was placed in an opaque plastic bucket $(57-\mathrm{cm}$ tall, $43-\mathrm{cm}$ diameter) filled with water $\left(21 \pm 2^{\circ} \mathrm{C}\right)$ to a $35-\mathrm{cm}$ depth for $10 \mathrm{~min}$ during the first week, $15 \mathrm{~min}$ during the second, and $20 \mathrm{~min}$ during third and fourth weeks. On completion, animals were dried and returned to the home cage. Social defeat was based on the residentintruder paradigm (Tornatzky and Miczek, 1994; Koolhaas et al., 1997). Ten male Long-Evans rats weighing around $150 \mathrm{~g}$ more than experimental subjects at the start of CUS were used as residents. To enhance territoriality, the resident was housed with a female Long-Evans rat until resident-intruder sessions. Some old bedding was always retained on cleaning residents' cages (weekly). The subject rat (intruder) was placed into the resident home cage, and they were allowed to interact until the intruder showed submissive supine posture or freezing or if $10 \mathrm{~min}$ had elapsed. During the first week, intruders were removed from the resident home cage and returned to their own home cage immediately following defeat (or $10 \mathrm{~min}$ ). During the second-fourth weeks, the resident was physically separated from the intruder following defeat by a wire-mesh partition and forced to remain in proximity to the resident for $30 \mathrm{~min}$ (second week) or $60 \mathrm{~min}$ (third and fourth weeks). To reduce habituation induced by repeatedly encountering the same resident, intruders confronted different residents in each session. Animals assigned to the control group were housed in groups of two or three and handled daily during the CUS period.

\section{Post-CUS Behavioral Measurements}

After CUS or control handling completion, all animals were housed in groups for 28 days (post-CUS period). On the day following the last CUS session, each animal was placed into the operant chamber and presented with active and inactive levers. They were retrained on an FR-1 schedule with the active lever to a criterion of 20 reinforcements in $30 \mathrm{~min}$. This session intended to assess effects of CUS on low-cost instrumental performance and to reinstate previously trained appetitive instrumental behavior. Performance on the PR schedule was tested on post-CUS Days 2, 11,19 , and 27. Tests were conducted under conditions similar to those for 3 daily PR sessions before CUS with ad libitum access to either water or food. On post-CUS Days 3 and 28, animals were placed in a NE for $30 \mathrm{~min}$. To preserve the novelty of the NE during repeated measurements, the configuration was changed before the second session (black dotted wall paper with sawdust bedding or black striped wall paper with paper-litter bedding, order counterbalanced across animals). Body weight was recorded weekly during both CUS and post-CUS periods.

\section{Serum Corticosterone Levels at Baseline and After Acute Stress Exposure}

In Experiment 2, morning blood samples (between 9:00 and 13:00) were collected from the tail vein for the measurement of resting state corticosterone concentration. These samplings were 
conducted firstly 2 days before CUS onset, secondly on Day 27 of the CUS period (= early post-CUS), and lastly on Day 25 of the post-CUS period (= late post-CUS) as depicted in Figure 3A. On the day before CUS Day 1 and on post-CUS Day 26, rats were placed individually in an electric-shock chamber $(40 \times 15 \times$ $40 \mathrm{~cm}$ ) equipped with an electrified grid floor (each grid bar: $0.4-\mathrm{cm}$ diameter, $1.6-\mathrm{cm}$ apart) for acute stress response testing. After a 5-min acclimation period, animals received 5 unavoidable electric footshocks $(40 \mathrm{~V}, 1 \mathrm{~s})$ with, on average, a 2-min intershock interval. Blood was collected from the tail vein $15 \mathrm{~min}$ after the last shock. Supernatants $(1000 \times g$ for $15 \mathrm{~min}$ and then $2000 \times g$ for $10 \mathrm{~min}, 4^{\circ} \mathrm{C}$ ) were stored at $-80^{\circ} \mathrm{C}$ until use. Corticosterone concentrations were determined using an ELISA kit (AssayPro, St. Charles, MO) according to the manufacturer's protocol.

\section{Statistical Analyses}

Hierarchical clustering to classify naïve animals based on PR instrumental performance (Experiment 1) was conducted using $\mathrm{R}$ ver. 2.15.3 software ( $\mathrm{R}$ Core Team, 2012). Between-group differences were tested using analyses of variance (ANOVA) and post hoc multiple comparisons with Ryan's procedure (Ryan, 1960). Two-Way or Three-Way repeated-measures ANOVAs were also used to analyze within-group repeated measures. Post hoc comparisons were performed by using simple-main or simple-simple-main effects tests and multiple comparisons with Ryan's procedure. Data points $\pm 2 S D$ from the subgroup mean were discarded in analysis of the set-shifting and reversal learning data. All statistical analyses were performed using ANOVA 4 on the Web (http://www.hju.ac.jp/ kiriki/anova4). Result reliability was assessed against a type I error $(\alpha)$ of 0.05 unless otherwise noted.

\section{Results}

\section{Classification of Individual Pre-stress Traits Based Instrumental Performance}

In Experiment 1 (Figure 1A), we trained a group of experimentally naïve rats to press an active lever on a timeconstrained ( $35 \mathrm{~min}$ ) PR schedule for 7 days. The mean number of lever presses declined progressively with each session, and significant differences were detected based on multiple comparisons between the first and third sessions, and between second and third sessions $\left[t \mathrm{~s}_{(156)}=4.38\right.$ and 2397 , respectively; Figure 1B]. In particular, considerable individual variability was observed on the first day. Therefore, we aimed to classify the animals based on two factors: (1) lever presses in the first PR session normalized by a common logarithm (log) transformation and (2) the change in the number of lever presses from the first to third sessions [change ratio $=(b-a) /(b+a)$, where $a$ and $b$ are the lever presses in the first and third sessions, respectively]. The performances of all animals fell within a relatively restricted area of a two-factorial graph (Figure 1C), and hierarchical clustering based on the Euclidean squared distances between data points defined four subgroups (Supplementary Figure 1): Cluster I, $n=17$ (21.5\% of all animals); Cluster II, $n=36$ (45.6\%); Cluster III, $n=13$ (16.5\%); and Cluster IV, $n=13$ (16.5\%). Cluster I performed significantly fewer lever presses in the first PR session than other three subgroups $\left[t_{(75)} \geq 9.64\right]$, whereas Cluster IV performed significantly more lever presses than Clusters II and III ( $t$ s $=8.23$ and 7.31, respectively: Figure 1D). Cluster III exhibited significantly lower change ratios than the other three subgroups $\left[t_{(75)} \geq 2.56\right]$, whereas Cluster IV exhibited significantly lower change ratios than Clusters I and II ( $t$ s $=4.09$ and 3.49, respectively; Figure 1E).

Next, we analyzed the subgroup differences in terms of their responses to active levers over seven PR sessions (Figure 1F; Cluster I, $n=13$; Cluster II, $n=32$; Cluster III, $n=12$; Cluster IV, $n=10$; the remaining animals were used for brain sampling, data not shown). In the third session, Cluster IV performed significantly more lever presses than the other three subgroups $\left[t_{(441)} \geq 3.24\right]$ and Cluster II performed significantly more lever presses than Clusters I and III ( $t \mathrm{~s}=4.85$ and 3.75 , respectively). Similarly, in the seventh session, Cluster IV performed significantly more lever presses than the other three subgroups ( $t \mathrm{~s} \geq 3.89$ ), and Cluster II performed significantly more lever presses than Clusters I and III ( $t \mathrm{~s}=3.72$ and 2.54, respectively). Similar subgroup differences were obtained for the inactive lever performance levels during the seven sessions (data not shown). In addition, we retrospectively analyzed subgroup differences in active lever presses during FR training, which preceded the PR training (Supplementary Figure 2). No significant subgroup differences were found during FR-1 training with water deprivation. However, significant subgroup differences emerged after water became accessible ad libitum. In the first session (FR-1) Cluster IV performed significantly more active lever presses than Clusters I and III $\left[t_{(300)}=3.78\right.$ and 2.92, respectively] and Cluster II performed significantly more lever presses than Cluster I $(t=2.89)$. Almost the same results were obtained from the second (FR-2) to the last (FR-5) sessions. In the last session, Cluster IV performed significantly more active lever presses than any of the other three subgroups $(t s \geq 7.09$ ) and Cluster I performed significantly fewer lever presses than Clusters II and III ( $t$ s $=4.41$ and 2.99 , respectively).

To identify the distinct behavioral and cognitive characteristics of the four subgroups, we calculated the individual change ratios in the response rates (lever presses/min) with the active lever between FR-1 training sessions with and without water deprivation (Supplementary Figure 2). This index (ad libitum water/with water deprivation) reflects the behavioral sensitivity to the change in the reward incentive value for a fluid reward ( $0.2 \%$ saccharin solution) because the incentive value was reduced by relieving water deprivation. As shown in Figure 2A, Cluster I had significantly lower change ratios than Clusters II and IV $\left[t_{(75)}=2.59\right.$ and 3.18] and Cluster III had significantly lower change ratios than Cluster IV $(t=2.78)$.

Based on the differences in instrumental performance with the PR schedule, the subgroups were designated as follows. Clusters I was designated as "Low Motivation" and Cluster IV as "Hypermotivation," because these subgroups performed the lowest and highest numbers of lever presses to obtain rewards during PR training, respectively. Cluster II was designated as "Quick Learner (QL)" because its members rapidly acquired a habitual action that was insensitive to changes in the reward 


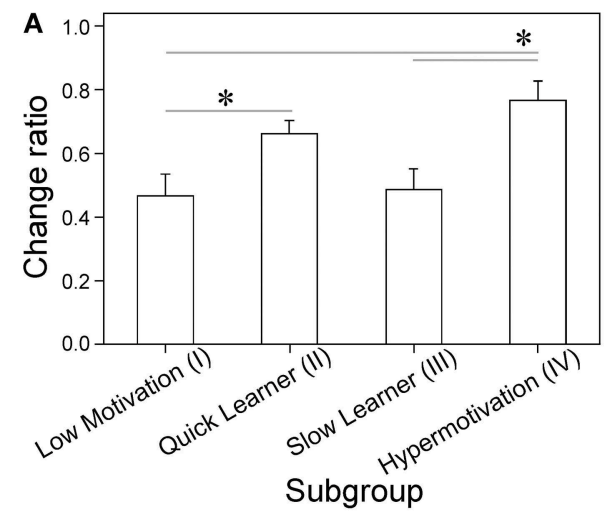

FIGURE 2 | Differential sensitivity to reward devaluation and variable number of errors during set-shifting of each subgroup. (A) Change ratios of active lever response rates (lever presses/min) with the fixed ratio reward schedule (FR-1) with vs. without water deprivation (index of sensitivity to devaluating the fluid reward); Cluster I, $n=17$; II, $n=36$; III, $n=13$; IV, $n=13$ [subgroup: $F_{(3,75)}=4.87, p=0.0038$ ]. (B) Analyses of three error types (perseverative, regressive, and never-reinforced) made during

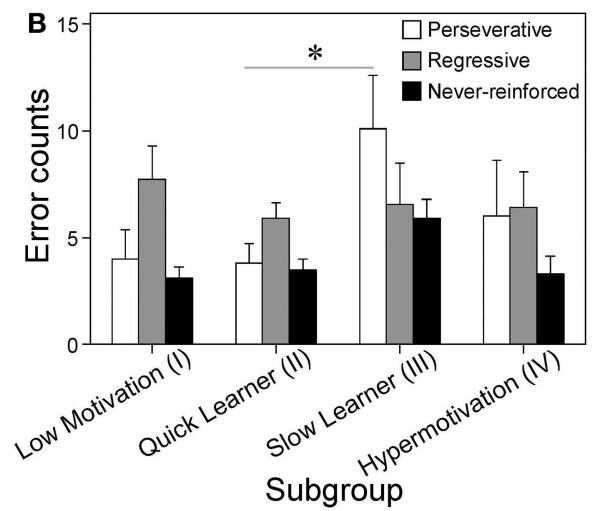

set-shifting by four subgroups. Data are shown as subgroup mean + SEM (data located outside mean $\pm 2 S D$ were discarded from the statistical analyses, changing the numbers of animals in each subgroups as follows; Cluster I, $n=11$; II, $n=27$; III, $n=11 ; \mathrm{IV}, n=7$ ), [subgroup on perseverative error: $F_{(3,52)}=3.04, p=0.037$; regressive error: $F_{(3,52)}<1$; never-reinforced error: $\left.F_{(3,52)}=3.02, p=0.038\right]$. ${ }^{*} p<0.05$, between relevant subgroups. value during PR training. Cluster III was designated as "Slow Learner (SL)," because its members still appeared to be sensitive to changes in the reward value compared with QL (Yin and Knowlton, 2006).

\section{Four Subgroups Exhibited Distinct Errors During Set-shifting}

We compared the performance in set-shifting and response reversal learning paradigms among the four subgroups. In set-shifting, the animals were first trained on a visual cue discrimination task where they chose the lever with an overhead light illumination cue. This training process progressed steadily without significant group differences (data not shown). In the subsequent set-shifting task, where the animals had to press the lever consistently while ignoring the visual cue, the SL subgroup made significantly more errors than the Low Motivation and QL subgroups $\left[t_{(52)}=2.84\right.$ and 4.10 , respectively; data not shown]. Analyses of error types showed that SL committed significantly more perseverative errors than QL $(t=2.90$ : Figure 2B). Significant effects of Subgroup were not found for regressive errors, whereas they were for never-reinforced errors $(p<0.05)$.

The animals were then trained by reversal learning, which first required the consistent choice of the previously rewarded (cued) lever (initial response discrimination) and then consistent choice of the other. This training also proceeded as expected without significant subgroup differences (data not shown). Similarly, None of subgroups were statistically significant in terms of the mean number of errors made during the subsequent response reversal learning as well (data not shown).

\section{Distinct Behavioral Responses of Subgroups to a Novel Environment and Acute Cocaine Administration}

There were no significant subgroup differences in the distance traveled (novel environment, NE) before cocaine administration
(Supplementary Figure 3A). However, because a Subgroup (4) $\times$ Time-block (24) ANOVA showed a marginal significant interaction between the factors $[F(69,874)=1.27, p=$ 0.076], we conducted subsequent simple-main effect tests and multiple comparisons. The Hypermotivation subgroup traveled a significantly greater distance than any of the other three subgroups in the third time-block after acute cocaine administration $\left[t_{(912)} \geq 3.21\right]$ and significantly greater distance than the QL subgroup in the second block $(t=2.89)$ and the Low Motivation subgroup in the fifth and seventh blocks ( $t$ s $=2.41$ and 2.52, respectively; Supplementary Figure 3B).

Supplementary Table 1 summarizes all of the distinct pre-CUS characteristics of the four subgroups.

\section{Distinct Subgroup Responses in Terms of Peripheral Corticosterone Levels and Body Weight Gain During and After Cus}

In Experiment 2, we aimed to determine whether the four subgroup classifications established in Experiment 1 could predict distinct responses to 4-week CUS (Figure 3A). Another group of experimentally naïve rats was trained to press an active lever with the PR schedule for three sessions in an identical manner to Experiment 1. According to the criteria established in Experiment 1, this second group was successfully divided into the four subgroups as defined above (Supplementary Figure 4). In brief, animals with $<1.5$ log-transformed active lever presses in the first PR session were classified as the Low Motivation subgroup (Cluster I, $n=27,32.1 \%$ of all animals) and those with $>2$ comprised the Hypermotivation subgroup (Cluster IV, $n=12,14.3 \%)$. Among the remainders, those with lever press change ratios that were greater or lesser than -0.2 between the first and third sessions were classified as the QL (Cluster II, $n=13,15.5 \%$ ) or SL (Cluster III, $n=32,38.1 \%$ ) subgroups, respectively. 


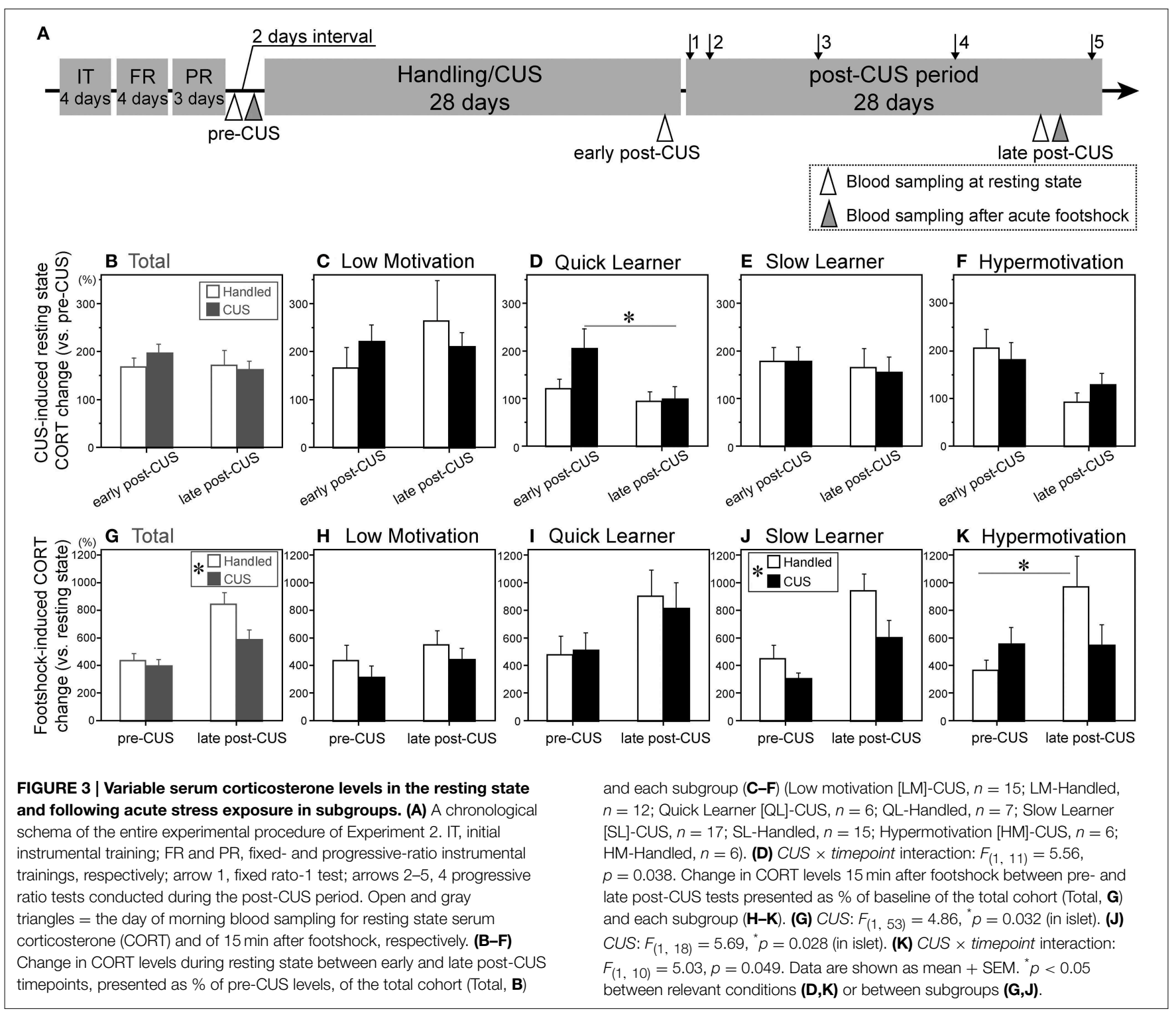

Each subgroup was then divided into control and CUSexposed (stressed) groups. After CUS, we compared the serum CORT levels at rest (Figures 3B-F) and $15 \mathrm{~min}$ after a footshock (Figures 3G-K) between control and stressed rats in each subgroup. The pre-CUS CORT at rest did not differ among the four subgroups (data not shown). In the total cohort, CUS had no effect on CORT at the early (CUS Day 25) or late post-CUS time point (post-CUS Day 27; Figure 3B). However, when they were classified into subgroups, there was a significant difference between early and late post-CUS in the stressed QL subgroup $\left[F_{(1,11)}=19.85\right.$; Figure 3D].

CUS reduced the sensitivity to the acute stressor based on the CORT increase in the total cohort (Figure 3G), SL (Figure 3J), and Hypermotivation (Figure 3K) subgroups. In the Hypermotivation subgroup, a significant difference in footshock-induced CORT between the two tests (pre- and late post-CUS) was observed only in handled animals $\left[F_{(1,10)}=9.83\right]$.

We assessed the effects of CUS on body weight gain during the CUS and post-CUS periods (Supplementary Figure 5). CUS altered body weight in the total group and all subgroups except for the Hypermotivation subgroup during the CUS period. In the total cohort, the effects of CUS on body weight appeared after 1 week and lasted until the end of the study. CUS had significant effects on the body weight on all days $\left[F_{(1,738)} \geq 7.18\right)$, except on CUS Day $1(F<1)$. In the Low Motivation subgroup, significant effects were found on all days $\left[F_{(1,225)} \geq 4.44\right]$, except on CUS Day 1 and post-CUS Day 22 ( $F$ s $\leq 3.85$ ). In the $\mathrm{QL}$ and SL subgroups, the effects were significant on all days $\left[F_{(1,99)} \geq 8.99\right.$ and $F s_{(1,270)} \geq 6.00$, respectively], except CUS Days 1 and 8 ( Fs $\leq 3.23$ ). However, in the Hypermotivation subgroup, the weight change was significant only on post-CUS Day $1\left[F_{(1,90)}=5.53\right]$. 


\section{Distinct Behavioral Reactions to a Novel Environment after CUS}

In Experiment 2, we examined the NE-induced behavior on postCUS Days 3 and 28. Overall, on both the early and late post-CUS days, the stressed animals exhibited enhanced novelty-induced locomotor activity (Figures $\mathbf{4 A}, \mathbf{F}$ ) compared with controls. The CUS effect on locomotor activity was significant only in the first 10-min blocks on both test days $\left[F_{(1,246)}=48.74\right]$. According to the subgroup analyses, CUS had effects on novelty-induced hyperlocomotion in the SL (Figures 4D,I) and Hypermotivation (Figures $4 \mathbf{E}, \mathbf{J}$ ) subgroups. In the SL subgroup, CUS had significant effects on locomotion only in the first 10min blocks on both test days $\left[F_{(1,90)}=22.10\right.$ : Figures $4 D$,I). In the Hypermotivation subgroup, CUS had a significant main effect $(p<0.05$; Figures 4E,J) but without significant interactions. In the Low Motivation (Figures 4B,G) and QL subgroups (Figures 4E,J), we found no main effects of CUS or interactions $(F \mathrm{~s} \leq 3.75)$.

\section{Distinct Short- and Long-term Effects of CUS on Appetitive Instrumental Performance}

In Experiment 2, we examined the instrumental lever pressing performance during the post-CUS period without water or food restrictions. On the day after the last CUS session, performance in low-cost instrumental learning (FR-1 schedule) was assessed (Figures 5A-E). Next, the, PR instrumental performance was assessed once each week (Figures $\mathbf{5 F}-\mathbf{J}$ ). In the FR-1 session, active lever pressing by the total cohort was accelerated by CUS [time-blocks $4-11$ and $14-48, F s_{(1,2460)} \geq 4.38$; Figure 5A]. Significantly enhanced lever pressing was also found in the Low Motivation [time-blocks 5-7, $F \mathrm{~s}_{(1,750)} \geq 5.21$; Figure 5B], and QL subgroups [time-blocks $3-19, F \mathrm{~s}_{(1,330)} \geq 4.16$; Figure 5C) but not in the SL and Hypermotivation subgroups (Figures 5D,E).

By contrast, CUS reduced the instrumental performance with the PR schedule in the total cohort both immediately after the CUS cessation [Day 2, time-blocks $21-35, F_{(1,14,350)} \geq 4.91$ ] and on Day 11 (time-blocks 15-35, Fs $\geq 4.12$ ) of the post-CUS period (Figure 5F). Reductions in the PR performance occurred in the QL [Day11. time-blocks 29-35, $F \mathrm{~s}_{(1,1925)} \geq 4$ 4.05; Figure $5 \mathrm{H}$ ] and Hypermotivation [regardless of test days, time-blocks 10$35, F s_{(1,350)} \geq 3.92$; Figure 5J] subgroups regardless of the test days. However, the Low Motivation subgroup did not exhibit significant changes in PR performance until about 3 weeks postCUS and on Day 27, the PR performance was significantly enhanced in stressed animals $\left[F_{(1,125)}=4.54\right.$ : Figure 5G]. In the SL subgroup, the main effects of CUS and the CUS-related interactions were not significant (Figure 5I).

All of these distinctive behavioral characteristics of the four subgroups are summarized in Supplementary Table 2.

\section{Discussion}

In this study, we classified naïe Sprague-Dawley rats into four subgroups by taking advantage of an instrumental learning with a PR schedule where the motivation for reward and the process of the instrumental learning could be assessed. The animals in each subgroup exhibited characteristic cognitive and behavioral traits at baseline as well as distinct behavioral responses to stress (CUS), although some of them are not involved as essential criteria for diagnosing MDD. Given the potential translational value of these results, we discuss possible relationships between the pre-CUS traits in rats and premorbid temperament in humans as well as between the post-CUS phenotypes in rats and MDD symptoms in humans.

\section{Practical Application of Subgroup Classification Based on PR Performance for Identifying Individual Differences at the Pre-stress Stage}

We classified the animals into behavioral subgroups (Low Motivation, QL, SL, and Hypermotivation) based on behavioral performance followed by hierarchical cluster analysis (Figures 1B-E, Supplementary Figure 1). In Experiment 1 , we found that individual differences in $\mathrm{PR}$ performance exceed those in FR performance (Supplementary Figure 2). PR-based classification was also sensitive to outcome devaluation (Figure 2A), errors made during set-shifting (Figure 2B), and cocaine-induced behaviors (Supplementary Figure 3). These data suggest that many behavioral and cognitive variables may be functionally related to each other and they can be segregated into small subgroups at pre-stress stage by PR performance.

\section{Cognitive and Behavioral Traits of Each Subgroup in the Pre-stress State}

Low Motivation animals were designated because of the least active lever presses during the first PR session, no further changes in the number of lever presses during the subsequent two sessions (Figures 1D-F), and significantly fewer active lever presses during the progression of the intermittent reinforcement schedule (Supplementary Figure 2A). Loss of motivation is one of the representative MDD symptoms. A similar phenotype is observed in rats with dopamine depletion in the nucleus accumbens, which is essential for motivation (Salamone and Correa, 2012). Although amotivation at premorbid state may appear odd, a recent human study suggests that adolescents with lower scores in the power and achievement motives may be vulnerable to MDD (Fuhr et al., 2014).

QL and SL subgroups were not distinguishable based on their active lever press rates during the first PR session (Figure 1D) and most of the FR sessions (Supplementary Figure 2A) but they could be differentiated by their change ratios (Figures 1E,F). The QL subgroup members also exhibited a different sensitivity to outcome devaluation compared with the SL subgroup (Figure 2A), thereby suggesting that the habitual process of instrumental learning was facilitated better in the QL subgroup than the SL subgroup (Yin and Knowlton, 2006).

We also found the lesser errors were made by the QL subgroup compared with by the SL subgroups in set-shifting (Figure 2B). Rapid adaptation to a newly introduced discrimination rule (reflected by fewer perseverative errors) appeared to be paralleled by rapid learning in this subgroup. QL was designated because of this general rapid learning property. Alternatively, it is possible that they are too impulsive to make action, which may result 
Early post-CUS
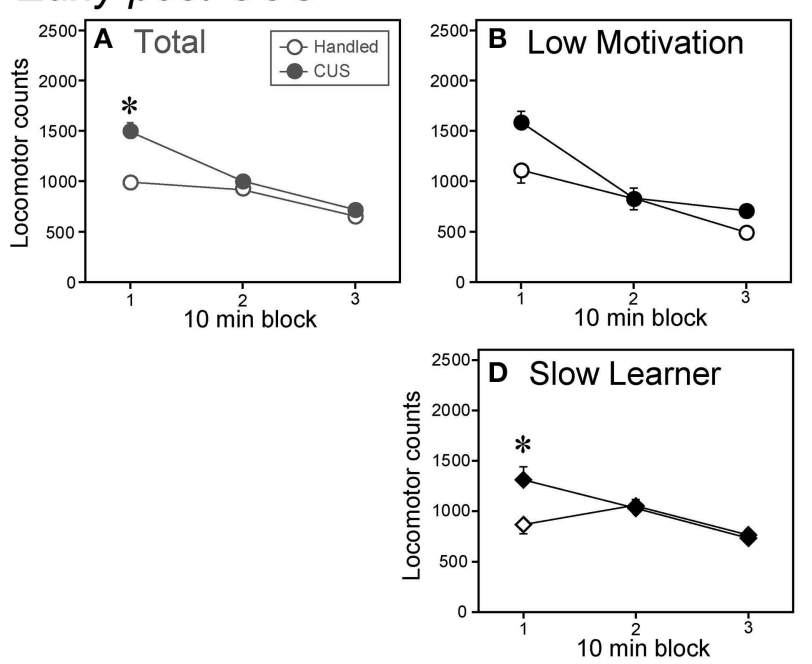

Late post-CUS
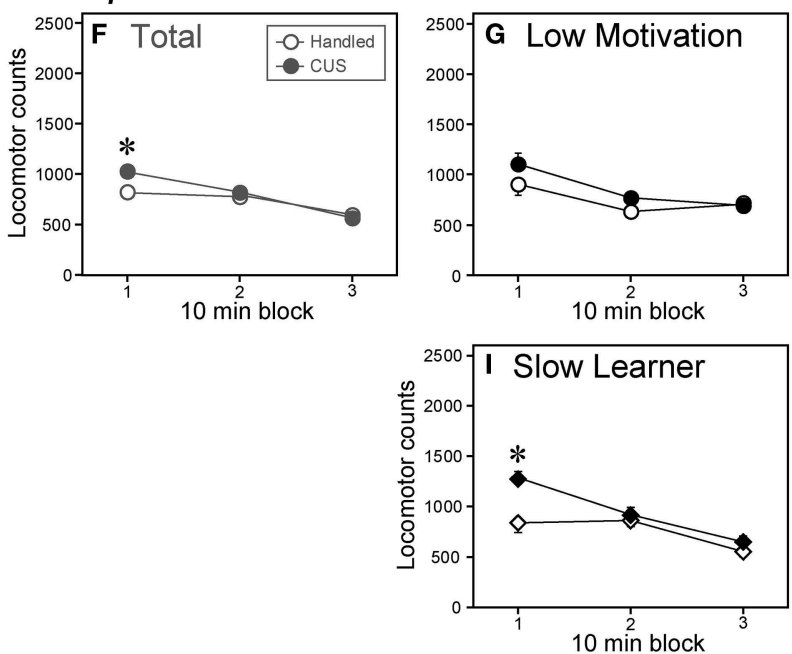
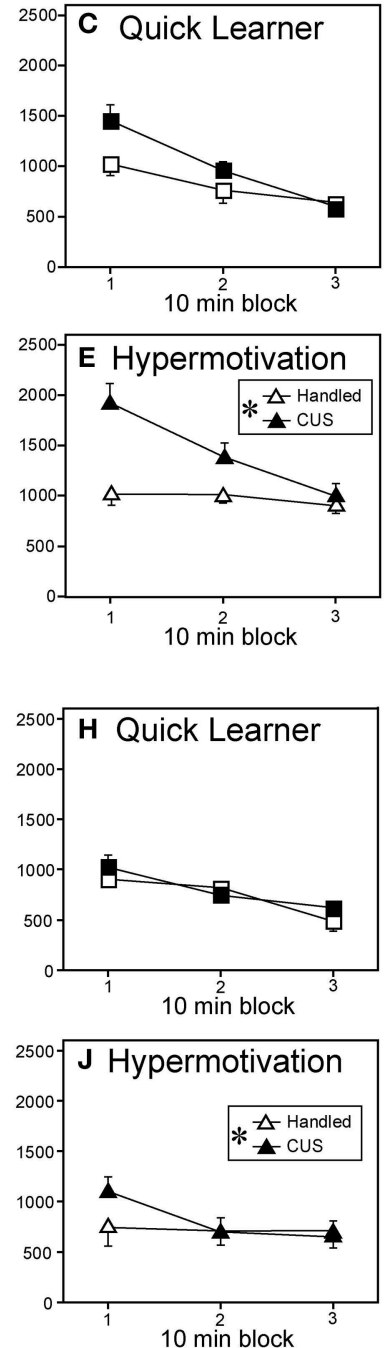

FIGURE 4 | Chronic unpredictable stress differentially affected locomotor reaction to a novel environment among subgroups. (A-E) Locomotor counts of CUS-exposed (filled symbols) and handled (open symbols) animals in a NE at Day 3 post-CUS (early post-CUS period). (F-J) Locomotor counts in a NE on Day 28 post-CUS (late post-CUS period). Data are shown as mean \pm SEM for the total cohort (Total, A,F) and each

subgroup (B-E and G-J; Low Motivation, circle; Quick Learner, square; Slow
Learner, lozenge; Hypermotivation, triangle. Low Motivation [LM]-CUS, $n=15$; LM-Handled, $n=12$; Quick Learner [QL]-CUS, $n=6$; QL-Handled, $n=7$; Slow Learner [SL]-CUS, $n=17$; SL-Handled, $n=15$; Hypermotivation [HM]-CUS, $n=6$; HM-Handled, $n=6)$. CUS $\times$ time-block interaction were $F_{(2,164)}=14.56, p<0.0001$ (A,F) and $F_{(2,60)}=9.38, p=0.0003$ (D,I). (E,J) CUS: $F_{(1,10)}=7.33,{ }^{*} p=0.022$ (in islet). ${ }^{*} p<0.05$, significant simple-main effect of CUS in a 10-min block or between handled and CUS. in a quick but premature learning. This idea is supported by the facilitation of habitual drug seeking by an impulsive predisposition (Everitt et al., 2008). By contrast, the SL subgroup exhibited the opposite trait in learning, namely greater cognitive persistence or slow learning. This subgroup comprised $16 \%$ of total, which appears to be excessively high to define a learning disability, so this subgroup may tend to be more prudent or perserverative (=less impulsive), and they may require numerous trials and errors to process new information or make decisions. In consistent with this, rats characterized at risk averse make more perseverative errors on set shifting (Shimp et al., 2015).
Finally, the Hypermotivation subgroup was designated because of the distinguished higher number of active lever presses than the others in the first PR session (Figures 1D,F) and a notable increase in active lever presses with the intermittent FR schedule (Supplementary Figure 2). During PR training, the reward provided was not accompanied by any caloric value or water/food deprivation, so the Hypermotivation subgroup was seeking excessively for a sweetness sensation and not for caloric value. Escalated novelty/sensation seeking is a characteristic feature of manic states and attention-deficit/hyperactive disorder (Hegerl et al., 2010), as well as addiction-prone individuals 

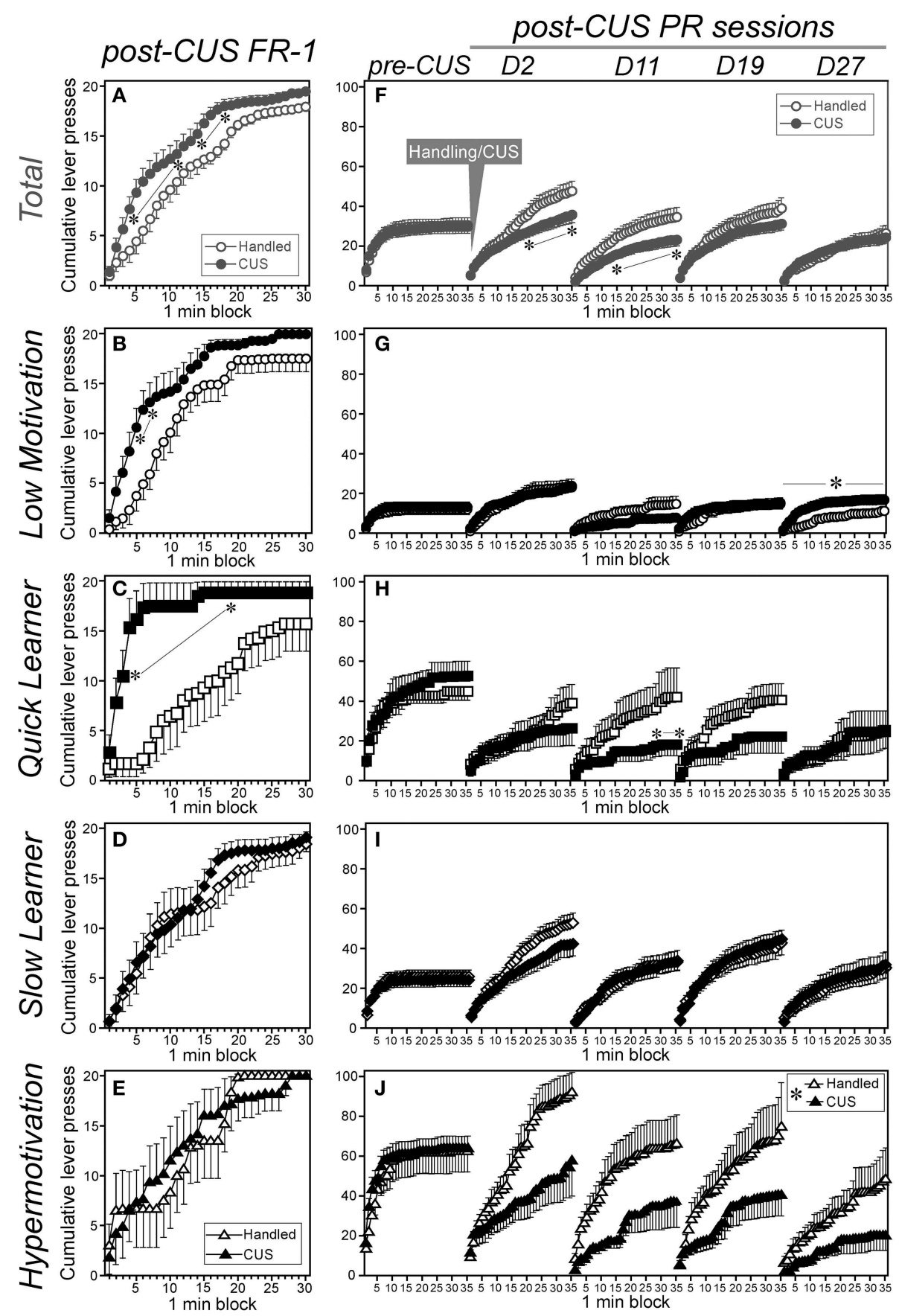

FIGURE 5 | Chronic unpredictable stress differentially affected constant low-cost or progressive ratio instrumental performance among subgroups. Cumulative saccharin-reinforced lever presses with the FR-1 (A-E; on the day following the last CUS session, left panels) or PR schedule (F-J; over time during the pre- and post-CUS periods, right panels) of CUS-exposed (filled symbols) and handled (open symbols) animals in the total cohort (A,F) and each subgroup (B,G: Low Motivation, circle; $\mathbf{C , H}$ : Quick Learner, square; D,I: Slow Learner, rhombus; E,J: Hypermotivation, triangle). Data are shown as mean \pm SEM (Low Motivation [LM]-CUS, $n=15$; LM-Handled, $n=12$; Quick Learner [QL]-CUS, $n=6$; QL-Handled, $n=7$; Slow Learner [SL]-CUS, $n=17$; SL-Handled, $n=15$; Hypermotivation [HM]-CUS, $n=6$; HM-Handled, $n=6$ ). CUS $\times$ time-block interaction were: (A) $F_{(29,2378)}=1.79, p=0.0060$, (B) $F_{(29,725)}=1.76, p=0.0083$, (C) $F_{(29,319)}=3.49, p<0.0001$, (F) $F_{(136,11,152)}=1.59, p<0.0001$, (G) $F_{(4,100)}=6.04, p=0.041,(\mathbf{H}) F_{(136,1496)}=1.42, p=0.0017$, and (J) $F_{(34,340)}=3.69,{ }^{*} p<0.0001$ (in islet). ${ }^{*} p<0.05$, significant simple-main effect of CUS in a 1-min block (A-C,F,H) or in the day (G), or between handled and CUS (J). 
(Steinberg, 2004; Cservenka et al., 2013). This pre-stress trait may correspond to hyperthymic temperament, which is one of the premorbid traits of bipolar disorder-like phenotypes in humans. Furthermore, this subgroup specifically exhibited an enhanced response to acute cocaine, another hallmark of the mania-like phenotype (Roybal et al., 2007, but see Hegerl et al., 2010; Supplementary Figure 3B).

\section{Distinct Effects of CUS on Each Subgroup PR Performance}

CUS induced loss of motivation to total cohort as shown in Figure 5F. The subsequent subgroup analyses revealed that this was mainly attributable to the Hypermotivation but partly to the QL subgroups (Figures 5H,J). In the Hypermotivation subgroup, although their motivation was still at the normal level from an objective viewpoint, they may have subjectively felt demotivated compared with their previous levels.

In case of the QL subgroup, the effect of CUS was transient and less robust effect compared with the Hypermotivation subgroup (Figure 5J). Alternatively, the natural rational process involved in reducing the active lever pressing may have been augmented in the stressed QL animals, which exhibited rapid adaptive learning as a pre-stress trait.

The SL subgroup displayed no effects of CUS throughout the entire post-CUS period in any respect, thereby implicating the most resistant to stress-induced demotivation among all.

Finally, the stressed Low Motivation animals began to press the active lever pressing more frequently than the controls following CUS from around post-CUS Day 27. In theory, active lever pressing should decrease gradually with the PR sessions, so this action appears to be unusual. However, because CUS favors habitual actions rather than flexible goal-directed actions (Dias-Ferreira et al., 2009), it is possible that an old habitual learning overwhelmed a recent flexible behavioral modification in a late-developing manner. Behavioral/cognitive inflexibility (or inadequate decision-making), which may result in extremely biased thoughts such as suicidal ideation, is one of the major hallmarks of MDD (Miranda et al., 2013). Further studies are necessary to address this issue.

\section{Body Weight}

In terms of body weight, CUS selectively affected the Low Motivation subgroup, thereby reflecting stress-induced appetite or metabolic suppression, whereas the Hypermotivation subgroup was almost resistant (Supplementary Figure 5).

\section{CORT}

Stress-induced elevation of CORT is considered to reflect the enhanced activity of hypothalamic-pituitary-adrenal (HPA) axis in MDD, although its functional significance is still debated (Kunzel et al., 2003; Lamers et al., 2013). At early post-CUS, CUS displayed its effect on CORT only in the QL subgroup in a temporal manner (Figure 3D). Given that habituation of the HPA-axis to repeated stress can function by attenuating the behavioral response to stress, such as general anxiety (Uchida et al., 2008), the QL subgroup may be resistant to CUS.
Meanwhile, CUS suppressed the acute footshock-induced increase in CORT in the SL and Hypermotivation subgroups (Figures 3J-K). However, the habituation to acute stress in these subgroups may lead to context- or object-specific anxiety, as reported in posttraumatic stress disorder (Santa Ana et al., 2006).

\section{Other Behavioral Indices}

CUS enhanced locomotor response in the NE in the SL and Hypermotivation subgroups (Figure 4). This behavioral augmentation may be functionally linked to the aforementioned blunted HPA-axis response to acute stress in these two subgroups, although the precise mechanism underlying it remains elusive.

Overall, our findings strongly suggest that distinct pre-CUS characteristics represented rather "traits" that were attributable to long-term functional interactions of various behavioral/cognitive properties, than merely "states" that reflected temporal biological or neural situations of each individual.

\section{Potential Advantages and Concerns}

As mentioned above, the limited effectiveness of antidepressants and the difficulty in predicting their effectiveness before medication are in part due to the current homogenous model of MDD. Thus, if the relationships between each subgroup with the response to different types of antidepressants can be clarified, more effective custom-made therapies could be formulated for each MDD individual.

It is still unclear whether the observed stress-response heterogeneity is based on genetic or epigenetic factors. In this study, we pooled two experimental sessions because it was difficult to test many animals simultaneously. Although all four subgroups were equally represented in both sessions, any differences in external factors, such as hierarchies among cage mates, transport conditions from a vendor, or any pre- or postnatal stress, may have been involved epigenetically (Fitzpatrick et al., 2013).

Another potential issue is the specificity of the CUS-induced phenotypes to MDD. Similar to MDD, onset or relapse is triggered by stress in many psychiatric disorders. In addition, many stress-induced symptoms such as depressive mood and loss of motivation are not specific to MDD. Therefore, it is possible that CUS may evoke phenotypes that are related to other mental disorders, particularly bipolar disorders. The identification of the hypermotivation subgroup as a pre-stress trait may support this possibility.

Finally, although this study could open another door to further understanding of MDD and its therapeutic approaches, as animal model of MDD it definitely needs to be validated with the response to antidepressants. Following studies employing antidepressants will be necessary.

In summary, we classified a group of experimentally naïve rats into four subgroups: Low Motivation, Quick Learner (QL), Slow Learner (SL), and Hypermotivation. We then clarified the effects of CUS on each subgroup. The Low Motivation subgroup exhibited lower motivation for the reward before CUS. After CUS, this subgroup exhibited weight loss and a 
TABLE 1 | A summary of causal relationships between putative pre-stress traits in humans that may correspond to pre-CUS traits in rats and putative post-stress MDD-related phenotypes that may correspond to post-CUS phenotypes in rats.

\begin{tabular}{|c|c|c|}
\hline Subgroup & $\begin{array}{l}\text { Putative pre-stress traits in humans corresponding } \\
\text { to pre-CUS traits in rats }\end{array}$ & $\begin{array}{l}\text { Putative post-stress symptoms in human MDD corresponding to post-CUS } \\
\text { phenotypes in rats }\end{array}$ \\
\hline Low motivation & Low motivation & Weight loss/Inflexible decision making \\
\hline Quick learner & Impulsivity & $\begin{array}{l}\text { Transient loss of motivation or enhanced adaptive behavior/Resistance to general } \\
\text { anxiety }\end{array}$ \\
\hline Slow learner & Prudency or Perseveration & Resistance to amotivation/Context- or object-specific anxiety \\
\hline Hypermotivation & Hyperthymia or Hyperactivity & $\begin{array}{l}\text { Long-lasting amotivation/Context- or object-specific anxiety/Resistance to weight } \\
\text { loss }\end{array}$ \\
\hline
\end{tabular}

Regular, pro-MDD-like, italic, anti-MDD-like traits or phenotypes.

late-developing paradoxical enhancement in PR performance which may be related to inappropriate decision-making in human MDD. The OL subgroup switched cognitive strategies rapidly at pre-CUS, which may reflect impulsivity. After CUS, this subgroup exhibited a transient loss of motivation (or rapid adaptive behavior) and the habituation of the HPA-axis response to repeated stress which may reflect resilience to general anxiety. The SL subgroup displayed prudency or persistency with respect to the initially acquired strategies at pre-CUS. After CUS, this subgroup exhibited resistance to demotivation and a suppressed HPA axis response to acute stress, so they may be prone to context- or object-specific anxiety. Finally, the Hypermotivation subgroup exhibited enhanced reward and sensation seeking as pre-CUS traits, which may correspond to hyperthymia/hyperactivity in humans. After CUS, this subgroup exhibited resistance to weight loss, habituated HPA response to an acute stress, and a long-lasting amotivation. It was notable that some of the CUS-induced phenotypes appeared to be anti-MDD-like rather than pro-MDD. The Low Motivation subgroup is probably the most vulnerable, whereas the QL and SL subgroups are relatively resistant to stress. Overall, distinct causal relationships between pre-stress traits and post-stress phenotypes were identified in each subgroup. Thus, the consequences of stress may be predicted before stress exposure by

\section{References}

Barr, A. M., and Phillips, A. G. (1998). Chronic mild stress has no effect on responding by rats for sucrose under a progressive ratio schedule. Physiol. Behav. 64, 591-597.doi: 10.1016/S0031-9384(98) 00060-2

Belin, D., Berson, N., Balado, E., Piazza, P. V., and Deroche-Gamonet, V. (2011). High-novelty-preference rats are predisposed to compulsive cocaine self-administration. Neuropsychopharmacology 36, 569-579. doi: 10.1038/npp.2010.188

Broos, N., Diergaarde, L., Schoffelmeer, A. N., Pattij, T., and De Vries, T. J. (2012). Trait impulsive choice predicts resistance to extinction and propensity to relapse to cocaine seeking: a bidirectional investigation. Neuropsychopharmacology 37, 1377-1386. doi: 10.1038/npp.2011.323

Collins, P. Y., Patel, V., Joestl, S. S., March, D., Insel, T. R., Daar, A. S., et al. (2011). Grand challenges in global mental health. Nature 475, 27-30. doi: $10.1038 / 475027$ a

Cservenka, A., Herting, M. M., Seghete, K. L., Hudson, K. A., and Nagel, B. J. (2013). High and low sensation seeking adolescents show distinct patterns identifying the pre-stress traits of each individual (summarized in Table 1).

\section{Author Contributions}

YI, SK, YM, and ST designed the experiments; YI, SK, ZL and HN performed all experiments; YI and ST analyzed data; YI, YM and ST wrote the manuscript.

\section{Acknowledgments}

This study was supported by Grants-in-Aids from the Ministry of Education, Culture, Sports, and Technology, Japan (20452097 and 25860994 to YI; 24791208 to SK; 26860920 to $\mathrm{HN}$; 25461727, 25116510, and 26120712 to ST), Eli Lilly (to YI), Japan Foundation for Neuroscience and Mental Health (to YI), Takeda Research Foundation (to ST), and Matsubara Saburoh Memorial Research Foundation for Psychiatry (to SK, ZL and YI).

\section{Supplementary Material}

The Supplementary Material for this article can be found online at: http://journal.frontiersin.org/article/10.3389/fnbeh. 2015.00119/abstract of brain activity during reward processing. Neuroimage 66, 184-193. doi: 10.1016/j.neuroimage.2012.11.003

Der-Avakian, A., and Markou, A. (2012). The neurobiology of anhedonia and other reward-related deficits. Trends Neurosci. 35, 68-77. doi: 10.1016/j.tins.2011.11.005

Dias-Ferreira, E., Sousa, J. C., Melo, I., Morgado, P., Mesquita, A. R., Cerqueira, J. J., et al. (2009). Chronic stress causes frontostriatal reorganization and affects decision-making. Science 325, 621-625. doi: 10.1126/science.11 71203

Duclot, F., and Kabbaj, M. (2013). Individual differences in novelty seeking predict subsequent vulnerability to social defeat through a differential epigenetic regulation of brain-derived neurotrophic factor expression. J. Neurosci. 33, 11048-11060. doi: 10.1523/JNEUROSCI.0199-13.2013

Everitt, B. J., Belin, D., Economidou, D., Pelloux, Y., Dalley, J. W., and Robbins, T. W. (2008). Review. Neural mechanisms underlying the vulnerability to develp compulsive drug-seeking habits and action. Philos. Trans. R. Soc. Lond. Biol. Sci. 363, 3125-3135.doi: 10.1098/rstb.2008.0089

Fitzpatrick, C. J., Gopalakrishnan, S., Cogan, E. S., Yager, L. M., Meyer, P. J., Lovic, V., et al. (2013). Variation in the form of Pavlovian conditioned 
approach behavior among outbred male Sprague-Dawley rats from different vendors and colonies: sign-tracking vs. goal-tracking. PLOS ONE 8:e75042. doi: 10.1371/journal.pone.0075042

Floresco, S. B., Block, A. E., and Tse, M. T. (2008). Inactivation of the medial prefrontal cortex of the rat impairs strategy set-shifting, but not reversal learning, using a novel, automated procedure. Behav. Brain Res. 190, 85-96. doi: 10.1016/j.bbr.2008.02.008

Fuhr, K., Hautzinger, M., and Meyer, T. D. (2014). Implicit motoves and cognitive variables: specific links to vulnerebility for unipolar or bipolar disorder. Psychiatry Res. 215, 61-68. doi: 10.1016/j.psychres.2013.10.001

Haluk, D. M., and Floresco, S. B. (2009). Ventral striatal dopamine modulation of different forms of behavioral flexibility. Neuropsychopharmacology 34, 2041-2052. doi: 10.1038/npp.2009.21

Harald, B., and Gordon, P. (2012). Meta-review of depressive subtyping models. J. Affect. Disord. 139, 126-140. doi: 10.1016/j.jad.2011.07.015

Hegerl, U., Himmerich, H., Engmann, B., and Hensch, T. (2010). Mania and attention-deficit/hyperactivity disorder: common symptomatology, common pathophysiology and common treatment? Curr. Opin. Psychiatry 23, 1-7. doi: 10.1097/YCO.0b013e328331f694

Koolhaas, J. M., De Boer, S. F., De Rutter, A. J., Meerlo, P., and Sgoifo, A. (1997). Social stress in rats and mice. Acta Physiol. Scand. Suppl. 640, 69-72.

Krishnan, V., Han, M. H., Graham, D. L., Berton, O., Renthal, W., Russo, S. J., et al. (2007). Molecular adaptations underlying susceptibility and resistance to social defeat in brain reward regions. Cell 131, 391-404. doi: 10.1016/j.cell.2007.09.018

Kunzel, H. E., Binder, E. B., Nickel, T., Ising, M., Fuchs, B., Majer, M., et al. (2003). Pharmacological and nonpharmacological factors influencing hypothalamicpituitary-adrenocortical axis reactivity in acutely depressed psychiatric inpatients, measured by the Dex-CRH test. Neuropsychopharmacology 28, 2169-2178. doi: 10.1038/sj.npp.1300280

Kurniawan, I. T., Guitart-Masip, M., and Dolan, R. J. (2011). Dopamine and effortbased decision making. Front. Neurosci. 5:81. doi: 10.3389/fnins.2011.00081

Lamers, F., Vogelzangs, N., Merikangas, K. R., De Jonge, P., Beekman, A. T., and Penninx, B. W. (2013). Evidence for a differential role of HPA-axis function, inflammation and metabolic syndrome in melancholic versus atypical depression. Mol. Psychiatry 18, 692-699. doi: 10.1038/mp.2012.144

McEwen, B. S. (2005). Glucocorticoids, depression, and mood disorders: structural remodeling in the brain. Metabolism 54, 20-23. doi: 10.1016/j.metabol.2005.01.008

Miranda, R., Valderrama, J., Tsypes, A., Gadol, E., and Gallagher, M. (2013). Cognitive inflexibility and suicidal ideation: mediating role of brooding and hopelessness. Psychiatry Res. 210, 174-181. doi: 10.1016/j.psychres, 0.2013.02.033

R Core Team. (2012). R: a Language and Environment for Statistical Computing. Available online at: http://www.r-project.org/

Richardson, N. R., and Roberts, D. C. (1996). Progressive ratio schedules in drug self-administration studies in rats: a method to evaluate reinforcing efficacy. J. Neurosci. Methods 66, 1-11. doi: 10.1016/0165-0270(95)00153-0

Roybal, K., Theobold, D., Graham, A., DiNieri, J. A., Russo, S. J., Krishnan, V., et al. (2007). Mania-like behavior induced by disruption of CLOCK. Proc. Natl. Acad. Sci. U.S.A. 104, 6406-6411. doi: 10.1073/pnas.0609625104

Rush, A. J. (2007). The varied clinical presentations of major depressive disorder. J. Clin. Psychiatry 68, 4-10.

Rush, A. J., Trivedi, M. H., Wisniewski, S. R., Nierenberg, A. A., Stewart, J. W., Warden, D., et al. (2006). Acute and longer-term outcomes in depressed outpatients requiring one or several treatment steps: a STAR ${ }^{\star} \mathrm{D}$ report. Am. J. Psychiatry 163, 1905-1917. doi: 10.1176/appi.ajp.163.11.1905
Russo, S. J., and Nestler, E. J. (2013). The brain reward circuitry in mood disorders. Nat. Rev. Neurosci. 14, 609-625. doi: 10.1038/nrn3381

Ryan, T. A. (1960). Significance tests for multiple comparison of proportions, variances, and other statistics. Psychol. Bull. 57, 318-328. doi: 10.1037/h00 44320

Rygula, R., Papciak, J., and Popik, P. (2013). Trait pessimism predicts vulnerability to stress-induced anhedonia in rats. Neuropsychopharmacology 38, 2188-2196. doi: 10.1038/npp.2013.116

Salamone, J. D., and Correa, M. (2012). The mysterious motivational functions of mesolimbic dopamine. Neuron 76, 470-485. doi: 10.1016/j.neuron.2012. 10.021

Santa Ana, E. J., Saladin, M. E., Back, S. E., Waldrop, A. E., Spratt, E. G., LaRowe, S. D., et al. (2006). PSTD and the HPA axis: differences in responses to the cold pressor task among individuals with child vs. adult trauma. Psychoneuroendocrinology 31, 501-509. doi: 10.1016/j.psyneuen.2005.11.009

Saunders, B. T., and Robinson, T. E. (2011). Individual variation in the motivational properties of cocaine. Neuropsychopharmacology 36, 1668-1676. doi: $10.1038 /$ npp. 2011.48

Shimp, K. G., Mitchell, M. R., Beas, B. S., Bizon, J. L., and Setlow, B. (2015). Affective and cognitive mechanisms of rsky decision making. Neurobiol. Learn. Mem. 117, 60-70. doi: 10.1016/j.nlm.2014.03.002

Stedenfeld, K. A., Clinton, S. M., Kerman, I. A., Akil, H., Watson, S. J., and Sved, A. F. (2011). Novelty-seeking behavior predicts vulnerability in a rodent model of depression. Physiol. Behav. 103, 210-216. doi: 10.1016/j.physbeh.2011. 02.001

Steinberg, L. (2004). Risk taking in adolescence: what changes, and why? Ann. N.Y. Acad. Sci. 1021, 51-58. doi: 10.1196/annals.1308.005

Tornatzky, W., and Miczek, K. A. (1994). Behavioral and autonomic responses to intermittent social stress: differential protection by clonidine and metoprolol. Psychopharmacology (Berl) 116, 346-356. doi: 10.1007/BF02245339

Trivedi, M. H., Rush, A. J., Wisniewski, S. R., Nierenberg, A. A., Warden, D., Ritz, L., et al. (2006). Evaluation of outcomes with citalopram for depression using measurement-based care in $\mathrm{STAR}^{\star} \mathrm{D}$ : implications for clinical practice. Am. J. Psychiatry 163, 28-40. doi: 10.1176/appi.ajp.163.1.28

Uchida, S., Nishida, A., Hara, K., Kamenoto, T., Suetsugi, M., Watanuki, T., et al. (2008). Characterization of the vulnerability to repeated stress in Fisher 344 rats: possible involvement of microRNA-mediated down-regualtion of the glucocorticoid receptor. Eur. J. Neurosci. 27, 2250-2261. doi: 10.1111/j.14609568.2008.06218.x

World Health Organization. (2014). Mental Disorder. Fact sheet $N^{\circ} 396$. Avaliable online at: http://www.who.int/mediacentre/factsheets/fs396/en/

Yin, H. H., and Knowlton, B. J. (2006). The role of the basal ganglia in habit formation. Nat. Rev. Neurosci. 7, 464-476. doi: 10.1038/nrn1919

Conflict of Interest Statement: We have received funding from two commercial sources, Takeda and Eli Lilly. However, we declare that they do not alter our adherence to all Frontiers policies on sharing data and materials. The authors declare that the research was conducted in the absence of ant commercial or financial relationships that could construed as a potential conflict of interest.

Copyright (C) 2015 Iguchi, Kosugi, Lin, Nishikawa, Minabe and Toda. This is an open-access article distributed under the terms of the Creative Commons Attribution License (CC BY). The use, distribution or reproduction in other forums is permitted, provided the original author(s) or licensor are credited and that the original publication in this journal is cited, in accordance with accepted academic practice. No use, distribution or reproduction is permitted which does not comply with these terms. 